\title{
Auditory temporal resolution in trained Indian classical dancers
}

\begin{abstract}
It's a well-known fact that conscious actions performed from auditory sensation involve activities in the central auditory nervous system. Previous literature reported impact of music in auditory processing. Previous literature showed enhanced auditory temporal resolution skill in musicians. Dance and music are related to each other, so it can be hypothesized that similar to musicians, dancer may also have enhanced auditory temporal resolution skill. There is a dearth of literature regarding auditory temporal resolution skill in dancers. Present study included 15 healthy Indian classical dancers (experimental group) and 15 healthy non-dancers (control group). To assess auditory temporal resolution skill in dancer and non-dancer, Gap Detection Test (GDT) and Duration Discrimination of Pure Tone (DDT) were used in the present study. The result revealed no significant difference in temporal resolution skill between dancers and non-dancers, which shows that Indian classical dance training has no substantial influence on auditory temporal resolution skill of the dancers
\end{abstract}

Keywords: Temporal resolution, dancers, Indian
Volume 10 Issue 2 - 2018

\author{
Himanshu Kumar Sanju,' Unnati Tayal' \\ 'Assistant Professor of Audiology and Speech Language \\ Pathology, Department of Audiology and Speech Language \\ Pathology, Amity Medical School,Amity University Haryana, India \\ 2Undergraduate student of Audiology and Speech Language \\ Pathology, Department of Audiology and Speech Language \\ Pathology, Amity Medical School, Amity University Haryana, India
}

Correspondence: Himanshu Kumar Sanju,Assistant professor of Audiology and Speech Language Pathology, Department of Audiology and Speech Language Pathology, Amity Medical School, Amity University Haryana, India, Tel +918447353649, Email himanshusanjuaiish@gmail.com

Received: February 01, 2018 | Published: March 09, 2018

\section{Introduction}

Earlier studies reported that brain structures differ between musicians and non-musicians. ${ }^{1}$ Previous literature reported that musicians have also reported better neuroplasticity among musicians compared to non-musicians. ${ }^{2}$ Earlier investigations among musicians concluded enhanced auditory evoked potential in musicians compared to non-musicians. ${ }^{3-5}$ Some of the studies also tried to tap auditory temporal resolution ability in musicians showed enhanced auditory temporal resolution in musicians compared to non-musicians. Similar to musicians, dancers also works with spatial and temporal resolution abilities, memory, cognition and motor coordination. Like musicians, dancers also continuously exposed to music. Other than just a physical activity, the dance includes sensory stimulation, emotions, motor coordination, social interaction and the music. Dance encourages extensive range of advantageous effects that are not restricted to motor development, posture and balance, but also comprises boosted cognitive skills.?

It's a well-known fact that conscious actions performed from auditory sensation involve activities in the central auditory nervous system. Previous literature reported impact of music in auditory processing. Previous literature showed enhanced auditory temporal resolution skill in musicians. ${ }^{5}$ Dance and music are related to each other, so it can be hypothesized that similar to musicians, dancer may also have enhanced auditory temporal resolution skill. There is a dearth of literature regarding auditory temporal resolution skill in dancers. It can be hypothesized that because dance is an activity that combines hearing and movement, dance practice could have positively changed important neural substrates for temporal processing, which could be demonstrated by test results of temporal resolution tests. The present study aimed to investigate auditory temporal resolution skill in Indian classical dancers compared to non-dancers. The objective of the study is to compare outcomes of Gap Detection Test (GDT) and Duration Discrimination Test (DDT) between Indian classical dancers (Bharatnatyam and Kathak) and non-dancers.

\section{Method}

Present study included 15 healthy Indian classical dancers (experimental group) and 15 healthy non dancers (control group). All the subjects participated in the study were having normal hearing revealed by pure tone audiometry, normal middle ear functioning revealed by tympanometry and reflexometry. All the participants were having normal outer hair cells functioning shown by oto-acoustic emission. Participants having any neurological and otological problem at the day of testing were excluded from the study. All the dancers participated in the present study were trained dancers in Indian classical dance (Bharatnatyam and Kathak) having a minimum experience of 4 years. All the dancers were regular practitioner of dance and they perform dance minimum 3-4 hours per week. All the dancers participated in the study, regularly attends stage shows at different events. The participants in control group (non-dancers) had not underwent any dance training neither they had performed any dance in any cultural activities. To assess auditory temporal resolution skill in dancer and non-dancer, Gap Detection Test (GDT) and Duration Discrimination of Pure Tone (DDT) were used in the present study. MATLAB software using maximum likelihood procedure was used to investigate these tests among dancers and non-dancers. Gap detection threshold was investigated using 750 milliseconds of Gaussian noise with gap in the center. Gap duration was varied according to subject performance using maximum likelihood procedure. The noise had 0.5 milliseconds cosine ramps at the beginning and end of the gap. In the three alternative forced choice task, the reference stimulus was always a 750-millisecond white noise without gap, whereas the variable stimulus contained a gap. We took the minimum gap duration required to perceive a gap in noise as the threshold. In duration discrimination using pure tone $(1000 \mathrm{~Hz})$, we measured the minimum difference in duration required to perceive the two otherwise identical stimuli, using maximum likelihood procedure. The duration of the standard tone was 250 milliseconds. The duration of the variable tone was changed based on response of the participants. We used two 
alternative forced choice procedures in which the participants were asked to indicate which tone was longer in duration. The collected data were tabulated in IBM SPSS statistics software (version 20) for further analysis of the data and to compare outcomes of tests between dancers and non-dancers.

\section{Results}

Descriptive statistics were done to find out mean, median and standard deviation of the threshold of GDT and DDT. Descriptive statistics revealed that mean threshold for GDT and DDT was smaller (better) in dancers compared to non-dancers. The mean threshold of GDT and DDT in dancers was $1.89 \mathrm{~ms}$ and $44.28 \mathrm{~ms}$ respectively. Whereas, the mean threshold of GDT and DDT in non-dancers was $2.02 \mathrm{~ms}$ and $45.99 \mathrm{~ms}$ respectively. The Figure $1 \& 2$ showed error bar graph of GDT and DDT respectively.

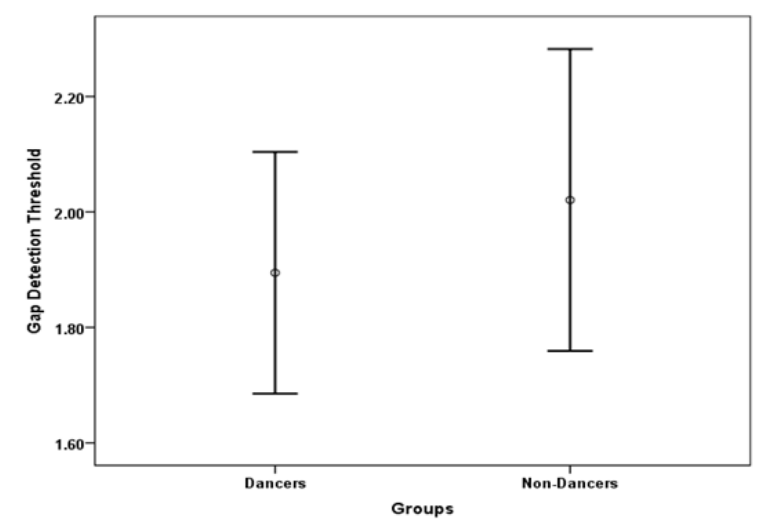

Figure I Gap Detection Threshold in dancers and non-dancers.

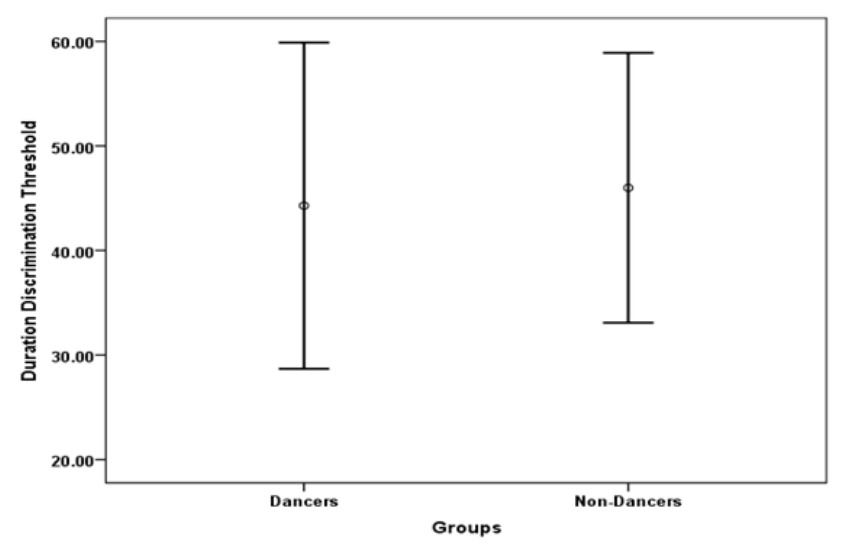

Figure 2 Duration Discrimination Threshold in Dancers and Non-Dancers.

Shapiro Wilk test showed normal distribution of data for GDT, whereas, non-normal distribution of data seen for DDT. Independent $\mathrm{t}$ test (parametric) showed no significant difference in GDT threshold between dancers and non-dancers $(\mathrm{t}=-0.80 ; \mathrm{df}=28 ; \mathrm{p}>0.05)$. Similarly, Mann Whitney U test showed (non-parametric test) also showed no significant difference in DDT threshold between dancers and nondancers.

\section{Discussion}

Dance provides an exceptional chance to study brain plasticity and its interaction with behavior. Previous investigation on dance observation recommend that long- and short-term dance training affect brain activity in the action observation and simulation networks. Despite methodological challenges, the feasibility of conducting neuroimaging while dancing has been demonstrated, and several brain regions have been implicated in dance execution. Present study revealed no significant difference in auditory temporal resolution ability between dancers and non-dancers which showed that Indian classical dance training has no effect on auditory temporal resolution ability of dancers. The present study is in contrast with the study done by Silva et al. ${ }^{8}$, where they reported better auditory temporal resolution ability in dancers compared to non-dancers. ${ }^{8}$ Small sample size could be the reason of no difference in auditory temporal resolution ability between dancers and non-dancers. Further studies should be carried out on large sample size to increase the validity of the study. In future, researchers can also investigate auditory temporal resolution skill on dancers of different variety of dance form.

\section{Conclusion}

Present study aimed to investigate temporal resolution skill in Indian classical dancers (Bharatnatyam and Kathak) compared to non-dancers. The result revealed no significant difference in temporal resolution skill between dancers and non-dancers, which shows that Indian classical dance training has no substantial influence on auditory temporal resolution skill of the dancers.

\section{Acknowledgement}

None.

\section{Conflict of interest}

None.

\section{References}

1. Gaser C, Schlaug G. Brain structures differ between musicians and non-musicians. J Neurosci. 2003;23(27):9240-9245.

2. Münte TF, Altenmüller E, Jäncke L. The musician's brain as a model of neuroplasticity. Nat Rev Neurosci. 2002;3(6):473-478.

3. Shahin A, Bosnyak DJ, Trainor LJ, et al. Enhancement of neuroplastic $\mathrm{P} 2$ and N1c auditory evoked potentials in musicians. $J$ Neurosci. 2003;23(13):5545-5552.

4. Kaganovich N, Kim J, Herring C, et al. Musicians show general enhancement of complex sound encoding and better inhibition of irrelevant auditory change in music: an ERP study. Eur J Neurosci. 2013;37(8):1295-1307.

5. Kumar P, Sanju HK, Nikhil J. Temporal Resolution and Active Auditory Discrimination Skill in Vocal Musicians. Int Arch Otorhinolaryngol. 2016;20(4):310-314.

6. Rammsayer T, Altenmüller E. Temporal information processing in musicians and nonmusicians. Music Perception: An Interdisciplinary Journal. 2006;24(1):37-48.

7. Kattenstroth JC, Kolankowska I, Kalisch T, et al. Superior sensory, motor, and cognitive performance in elderly individuals with multiyear dancing activities. Front Aging Neurosci. 2010;24(5):63-69.

8. Silva MR, Dias KZ, Pereira LD. Study of the auditory processes of temporal resolution and auditory figure-ground in dancers. Revista Cefac. 2015;17:25-29. 